B Research Square $=$

\title{
A novel radiographic grid aiming device for percutaneous renal puncture
}

Guibin Xu ( $\nabla$ uro_xgb@163.com )

Fifth Affiliated Hospital of the Guangzhou Medical University

Research article

Keywords: renal puncture, bull's eye technique, PCNL, papillary fornix

Posted Date: September 14th, 2021

DOI: https://doi.org/10.21203/rs.3.rs-885643/v1

License: (a) (i) This work is licensed under a Creative Commons Attribution 4.0 International License.

Read Full License 


\section{Abstract \\ Background}

This in-vitro study aims to assess the efficacy of a novel radiographic grid aiming device as compared to the conventional "bull's eye" puncture technique for percutaneous renal puncture.

\section{Methods}

This new device uses two radiopaque rings for targeting, somewhat similar to the concept of gun sights. The device is designed to improve the ease and accuracy of the "bull's eye" puncture technique. We compared this device to the conventional "bull's eye" puncture technique using a porcine kidney inside a chicken carcass as the study model. Two first-year residents used both the radiographic grid aiming device in turn, Group A, and the conventional bull's eye technique, Group B, to perform punctures to reach the target porcine calyces in order. Related data were recorded and analyzed.

\section{Results}

23 renal calyceal punctures were performed per group. There were no statistical differences in the overall puncture success rate, puncture time, or radiation exposure time and dose between the two groups. The number of punctures required to achieve success was higher in group $B$ and the distance between the puncture site to the center of papillary fornix was shorter in group $A$, all $P<0.05$.

\section{Conclusions}

The novel radiographic grid aiming device showed no advantage in the overall success rate for renal puncture. However, it offered the advantages of more precise puncture with fewer puncture attempts. It may serve as a useful tool for training. It may also offer some benefit to surgeons who are novices at percutaneous renal puncture.

\section{Background}

Percutaneous nephrolithotomy (PCNL) is the commonly accepted treatment method for larger renal stones. Precise calyceal puncture is the first and key step in PCNL. An ideal calyceal puncture should ensure the least trauma for nephrostomy tract creation, complete removal of the calculi, and minimal complications. The puncture should be aimed at the center of the papillary of the targeted calyx.

Conversely, an inaccurate puncture may result in injuries to the kidney and excessive bleeding, and thus impact the overall surgical success and patient outcome. The "bull's eye" technique usually is the basic technique taught to novices [1]. Any of the percutaneous puncture techniques generally has a fairly steep learning curve. One of our guest faculty, Professor Shaw P. Wan, M.D., developed a novel puncture device 
using two radiographic grids with same-size radiopaque targeting rings for aiming the puncture needle. Professor Wan has asked us at the Translational Laboratory of the Fifth Affiliated Hospital of the Guangzhou Medical University to independently evaluate this device. We feel that this device may improve the accuracy of percutaneous renal puncture and shorten the learning curve; therefore, we consented to undertake this task. We used a porcine kidney inside a whole chicken carcass as the study model for this experiment.

\section{Materials And Methods}

\section{Preparation of ex vivo porcine kidney in chicken carcass model}

This study was approved by the Ethics Committee of the Fifth Affiliated Hospital of the Guangzhou Medical University. Whole chickens were purchased from the supermarket. They were completely disemboweled and cleaned to ensure adequate space for insertion of porcine kidneys. The porcine kidneys with ureters five centimeters or longer were purchased directly from the slaughterhouse. Any kidney with anatomical abnormality or obvious hydronephrosis was discarded. The ureter was carefully dissected free from the surrounding adipose tissue. The renal capsule was stripped to remove any fluid that might extravasate between the kidney and the capsule during the puncture trials. The accumulated fluid might give a false notion of reaching the targeted calyx. A five French ureteral catheter was inserted into the renal pelvis through the ureter and tied in place. The porcine kidney was then placed inside the chicken carcass with the chicken in a supine position (the abdomen of the chicken faced upward). The kidney, however, was rotated to a position that mimicked the kidney in a human body in prone position. Radiolucent sponges were used to fix the kidney in place when necessary. The skin of the chicken was closed with silk suture to complete the study model (Figure 1).

\section{Radiographic grid aiming device}

The device consists of two circular radiographic grids. The grids are constructed with radiolucent material with radiopaque centrally located rings. Thus, the radiographic images of the grids show mostly the central rings. The grids are designated as proximal and distal grids. The distal grid is placed on the surface of the object to be punctured. The proximal grid is held near the image intensifier of the fluoroscopy machine. Both grids and their central rings are the same size. There is also a central aperture in each of the grids for passage of the puncture needle. The distal grid is constructed with soft silicone material. The distal grid aperture allows the passage of the puncture needle. The proximal grid is made of hard clear plastic. The proximal grid aperture is constructed with a luer lock mechanism to allow the puncture needle to be detachably secured to the proximal grid. In addition, the proximal grid has peripheral direction markers to aid in aligning the grids. A handle can be attached to the proximal ring so that the operator's hand can be outside the imaging field during the puncture (Figure 2). On the fluoroscopic image, the distal ring appears to be smaller than the proximal ring. However, as the puncture advances through tissue, i.e. the chicken and porcine kidney, both rings begin to approximate each other's sizes. 


\section{Comparison of the two puncture techniques}

The study was conducted by two first-year residents (Xu, Zhu) who had minimal exposure to PCNL and had not performed any percutaneous puncture on actual patients. All the procedures were performed using C-arm fluoroscope by two residents, randomly. 46 qualified chicken-porcines were divided into two groups of equal numbers and performed by two first-year residents in turn. Group A models were punctured using the radiographic grid aiming device. Group B used the conventional "bull's eye" technique. Contrast material mixed with methylene blue was injected into the porcine kidney in retrograde fashion to image the pyelocalyceal system as well as to create a mild artificial hydronephrosis. The most direct anterior upper pole calyx was selected as the target.

\section{3-1 Puncture using the radiographic grid aiming device-Group A}

The distal grid was placed on the abdomen of the chicken with the central ring over the targeted calyx. Static images were used for adjusting the position of the grid. A modified two-part trocar puncture needle (with luer lock connection) was secured to the proximal grid through the locking mechanism. The handle was then attached to keep the operator's hand outside the imaging field. The needle was inserted through the central aperture of the distal grid. Using continuous fluoroscopic images, the two central rings were aligned, and the needle was advanced. This was accomplished with the C-arm in an approximately $30^{\circ}$ angle. After about two centimeters of advancement, the fluoroscope was moved to $0^{\circ}$ away from the operator to check the depth of penetration (Figure 3). Successful puncture of the targeted calyx was defined by first observing the previously-instilled contrast material draining through the needle hub, followed by injection of fresh contrast material through the needle for further image confirmation. Next, a $0.035 "$ guide wire was passed through the puncture needle and advanced down the ureter. The puncture needle was removed to finish the experiment. The time required for a successful puncture, number of trials to achieve a successful puncture, and fluoroscopy exposure time and dose were recorded. Failure was defined by failing to reach the selected calyx after six attempts. The successfully punctured porcine kidney was next retrieved with the guide wire in place and dissected to expose the punctured calyx. The distance between the needle puncture site and the center of the calyceal was measured and recorded. We used a scoring system to define the distance between the needle entry point and the center of renal papilla (NEP-CRP Score). If the distance was between 0 and $1 \mathrm{~mm}$, we gave a of score 1 ; for $>1 \mathrm{~mm}$ to $2 \mathrm{~mm}$, we gave a score of 2 ; and so on; thus, $>8 \mathrm{~mm}$ to $9 \mathrm{~mm}$ would have a score of 9 .

\section{3-2. Conventional bull's eye puncture technique -control}

An 18-gauge standard two-part trocar puncture needle was used. A long straight Kelly clamp was used to hold the needle shaft and keep the operator's hand outside the imaging field. The tip of the needle was placed directly over the targeted calyx using static and fluoroscopic images. The needle was then rotated $90^{\circ}$ so that it was now in a straight line with the image receptor to create a "bull's eye" image. The needle was advanced downward under continuous fluoroscopic images. After about two centimeters of advancement, the fluoroscope was angled to $0^{\circ}$ away from the operator to check the depth of 
penetration. Successful puncture of the targeted calyx was similarly defined by first observing the previously instilled contrast material draining through the needle hub followed by injection of fresh contrast material through the needle for image confirmation. Failure to reach the target after six attempts was deemed failure. Relative data were recorded. The porcine kidney was then retrieved and the distance between the needle puncture site and the center of the calyceal was measured and recorded.

\section{Statistical analysis}

Statistical analysis was performed using the SPSS version 19.0. software. Continuous variables were analyzed using parametric tests. $P<0.05$ was deemed statistically significant.

\section{Results}

46 porcine kidneys were punctured. As shown in Table 1, there was no statistical difference in the overall success for punctures between Group A and Group B ( $77.8 \%$ versus $83.3 \% ; P=0.457)$. There were also no statistical differences in the puncture time or radiation time and dose for successful puncture between the two groups $(P>0.05)$. However, the number of punctures required to achieve success was higher in group $\mathrm{B}(P=0.01)$, and the distance from the puncture site to the center of papillary (the NEP-CRP Score) was shorter in group $A$.

\section{Discussion}

PCNL is a demanding endourological procedure. Precise puncture is the first step and one of the most crucial steps in PCNL. It can be challenging even in the most experienced hands. The puncture can be performed under either fluoroscopic, ultrasonographic, or combined guidance. Currently, fluoroscopicguided puncture remains the mainstay technique. The most commonly used fluoroscopic-guided puncture techniques are the "bull's eye" and the triangulation techniques [1, 2]. Precise percutaneous renal puncture has a fairly steep learning curve; thus, it deters many urologists from performing this procedure. The radiographic grid aiming device has been developed with the goals to improve the accuracy of renal puncture and to shorten the learning curve.

Whether papillary puncture is necessary for PCNL is still being debated [3-6]. Renal access through the center of the papilla is thought to be ideal. Reasons for papillary puncture include the assumption that it is the shortest tract through the renal parenchyma $[5,7,8]$ and anatomical study suggests that punctures through the papillary pose the least injury to the kidney and its vasculature [9].

Historically, renal access for PCNL has been performed mostly by interventional radiologists. According to the literature, $69.7 \%$ of urologists perform PCNL, but only $11 \%-27 \%$ obtain their own access despite training in percutaneous surgery during their residencies $[10,11]$. Evidence has shown that percutaneous access performed by urologists themselves is associated with a statistically significant improvement in the overall stone-free rate $[12,13]$. Published literature suggests that the learning curve for PCNL is about 60 cases to derive competence $[14,15]$. The novel radiographic grid aiming device may shorten this 
learning curve, as proven by the fact that even in inexperienced hands, a stable and precise outcome can be achieved (Coefficient of Variation for NEP-CRPF Score: 0.393 vs. 0.521 for Group A and Group B respectively).

The weaknesses of this study include that it is a single institution study. Moreover, Professor Wan is one of our guest faculty. Even though there is no financial conflict of interest, there is the potential of unintentional bias favoring him. Further studies may be beneficial once this device is commercialized.

\section{Conclusion}

The radiographic grid aiming device offers the advantages of more precise puncture and fewer puncture trials to achieve successful puncture. It may serve as a useful training tool. It may also offer some benefits to surgeons who are novices at percutaneous renal puncture.

\section{Abbreviations}

PCNL

Percutaneous nephrolithotomy

NEP-CRP

the needle entry point and the center of renal papilla

\section{Declarations}

\section{Ethics approval and consent to participate}

This article does not contain any studies with human participants or animals performed by any of the authors.

\section{Funding}

High-level university academic backbone training program of Guangzhou Medical University

\section{Authors' Contributions}

Y.X., R.Z. and Z.O. were contributed equally to project development, data collection and management and article writing/editing. Y.Y. and S.C. contributed to protocol/project development data collection and management. X.L. contributed to data collection and analysis, and article writing/editing. G.X. contributed to protocol/project development.

\section{Authors' Information}

Department of Urology, the Fifth Affiliated Hospital of the Guangzhou Medical University, No 621, Gangwan Road, Huangpu District, Guangzhou, 510700, China; 
Yuyu Xu ${ }^{1, *}$, Rui Zhu ${ }^{1, *}$, Yaoji Yuan, Xiezhao Li, Shuangxing Chen, Guibin Xu

Department of Radiology, the Fifth Affiliated Hospital of the Guangzhou Medical University, No 621, Gangwan Road, Huangpu District, Guangzhou, 510700, China;

Zhongmin Ouyang ${ }^{2, *}$

* Yuyu Xu, Rui Zhu and Zhongmin Ouyang contributed equally to this work and are listed as the co-first authors.

\# Corresponding author: Guibin Xu, Address: No 621, Gangwan Road, Huangpu District, Guangzhou, 510700, China; Tel: 13802432800; e-mail: gyxgb@163.com

\section{Consent for publication}

Not applicable.

\section{Competing interests}

The authors declare no competing interests.

\section{Acknowledgements}

We fortunately have Professor Wan to serve as a guest faculty. Professor Wan is a retired American urologist. He has returned to his ancestral home to perform community services and as a voluntary teaching staff at several hospitals. His generous efforts have greatly improved our clinical skills and patient care.

\section{References}

1. Tepeler A,Binbay M,Yuruk E,Sari E,Kaba M,Muslumanoglu AY, Tefekli A (2009) Factors affecting the fluoroscopic screening time during percutaneous nephrolithotomy. Journal of endourology 23(11):1825-1829.

2. Miller NL,Matlaga BR, Lingeman JE (2007) Techniques for fluoroscopic percutaneous renal access. The Journal of urology 178(1):15-23.

3. Tahra A,Sobay R,Bindayi A,Suceken FY, Kucuk EV (2020) Papillary vs non-papillary access during percutaneous nephrolithotomy: Retrospective, match-paired case-control study. Archivio italiano di urologia, andrologia : organo ufficiale [di] Societa italiana di ecografia urologica e nefrologica 92(1):5052 .

4. Kallidonis P, Liatsikos E (2019) Puncture for percutaneous surgery: is papillary puncture a dogma? Yes! Current opinion in urology 29(4):470-471. 
5. Sampaio FJ (2000) Renal anatomy. Endourologic considerations. The Urologic clinics of North America 27(4):585-607, vii.

6. Kallidonis P,Vagionis A,Vrettos T,Adamou K,Pagonis K,Ntasiotis P,Callas GA,Tanaseskou L,Al Aown AM, Liatsikos E (2020) Non papillary mini-percutaneous nephrolithotomy: early experience. World journal of urology.

7. Kallidonis P,Kyriazis I,Kotsiris D,Koutava A,Kamal W, Liatsikos E (2017) Papillary vs Nonpapillary Puncture in Percutaneous Nephrolithotomy: A Prospective Randomized Trial. Journal of endourology 31(S1):S4-s9.

8. Sampaio FJ, Aragao AH (1990) Anatomical relationship between the intrarenal arteries and the kidney collecting system. The Journal of urology 143(4):679-681.

9. Sampaio FJ,Zanier JF,Aragão AH, Favorito LA (1992) Intrarenal access: 3-dimensional anatomical study. The Journal of urology 148(6):1769-1773.

10. Lee CL,Anderson JK, Monga M (2004) Residency training in percutaneous renal access: does it affect urological practice? The Journal of urology 171(2 Pt 1):592-595.

11. Vicentini FC,Gomes CM,Danilovic A,Neto EA,Mazzucchi E, Srougi M (2009) Percutaneous nephrolithotomy: Current concepts. Indian journal of urology : IJU : journal of the Urological Society of India 25(1):4-10.

12. El-Assmy AM,Shokeir AA,Mohsen T,El-Tabey N,El-Nahas AR,Shoma AM,Eraky I,El-Kenawy MR, ElKappany HA (2007) Renal access by urologist or radiologist for percutaneous nephrolithotomy-is it still an issue? The Journal of urology 178(3 Pt 1):916-920; discussion 920.

13. Watterson JD,Soon S, Jana K (2006) Access related complications during percutaneous nephrolithotomy: urology versus radiology at a single academic institution. The Journal of urology 176(1):142-145.

14. Sahan M,Sarilar O,Savun M,Caglar U,Erbin A, Ozgor F (2020) Adopting for Supine Percutaneous Nephrolithotomy: Analyzing the Learning Curve of Tertiary Academic Center Urology Team. Urology 140:22-26.

15. Tanriverdi O,Boylu U,Kendirci M,Kadihasanoglu M,Horasanli K, Miroglu C (2007) The learning curve in the training of percutaneous nephrolithotomy. European urology 52(1):206-211.

\section{Tables}

Table 1: Data for successful puncture between Group A and B 


\begin{tabular}{llll}
\hline Item & $\begin{array}{l}\text { Group A } \\
(\mathrm{N}=20)\end{array}$ & $\begin{array}{l}\text { Group B } \\
(\mathrm{N}=17)\end{array}$ & $\begin{array}{l}\text { Value of } \\
P\end{array}$ \\
\hline Overall success rate (success/failure) & $20 / 3$ & $17 / 6$ & 0.46 \\
Puncture time (seconds) & $278.20 \pm 94.63$ & $445.64 \pm 308.01$ & 0.07 \\
Number of punctures & $1.47 \pm 0.64$ & $2.21 \pm 0.80$ & $0.01^{*}$ \\
Number of static X-ray & $22.79 \pm 14.98$ & $18.60 \pm 6.13$ & 0.35 \\
Fluoroscopic exposure time & $13.97 \pm 4.62$ & $17.08 \pm 12.74$ & 0.40 \\
Fluoroscopic dose (mGy) & $0.50 \pm 0.19$ & $0.77 \pm 0.49$ & 0.06 \\
NEP-CRPF Score & $3.80 \pm 1.32$ & $5.0 \pm 2.12$ & $0.04 *$ \\
Coefficient of Variation (SD/Mean) for the NEP-CRP & 0.347 & 0.424 & - \\
Score & & & \\
\hline
\end{tabular}

Group A = the group of the radiographic grid aiming device; Group B = the group of the conventional bull's eye technique; NEP-CRP Score = the distance between the needle entry point and the center of renal papilla fornix;

\section{Figures}




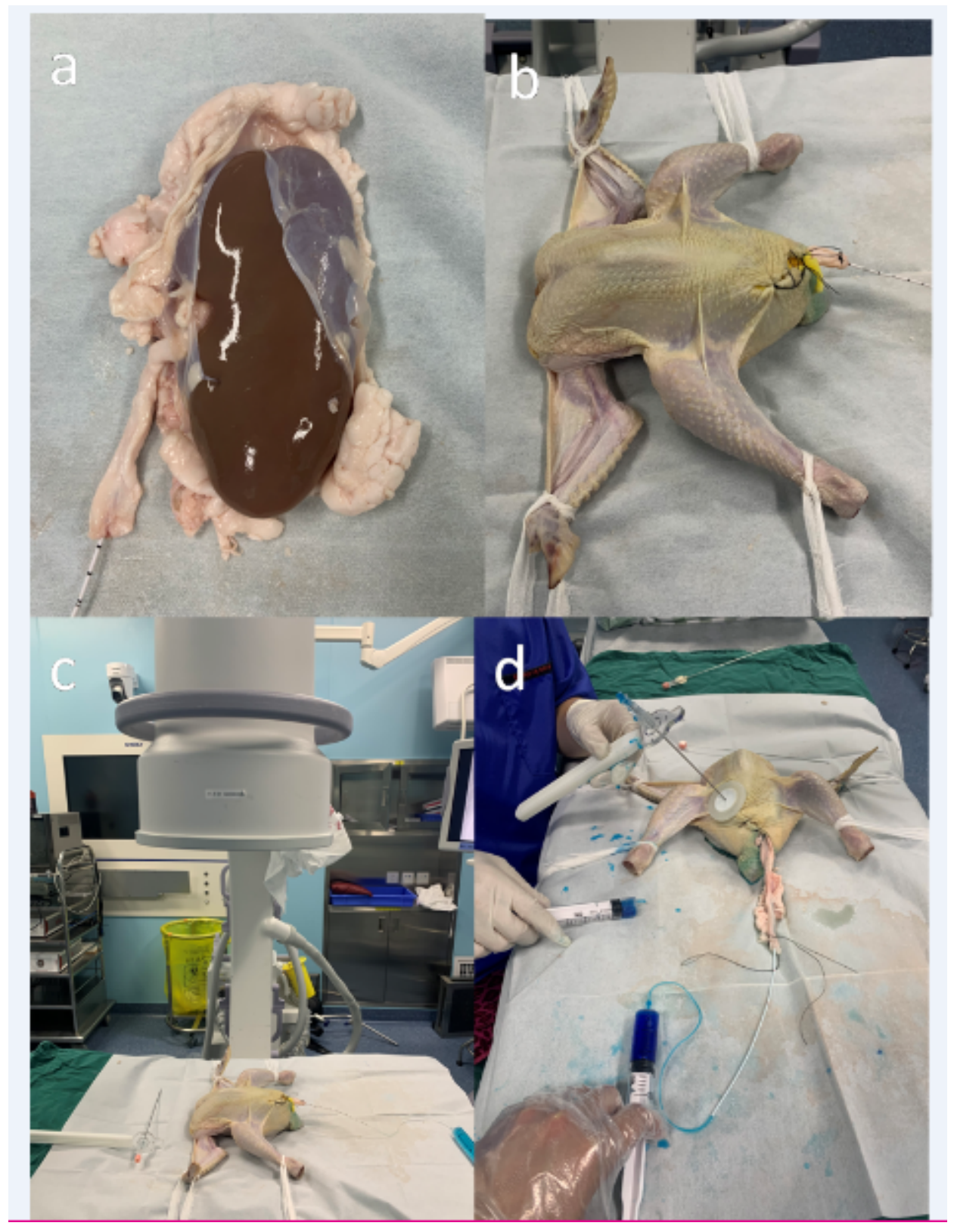

\section{Figure 1}

Study Model: (a) Renal capsule is removed from porcine kidney. $5 \mathrm{~F}$ ureteric catheter is placed into the ureter and the renal pelvis. (b) The chicken is fixed to the operating table. (c, d) C-arm and instruments used in the experiments. 

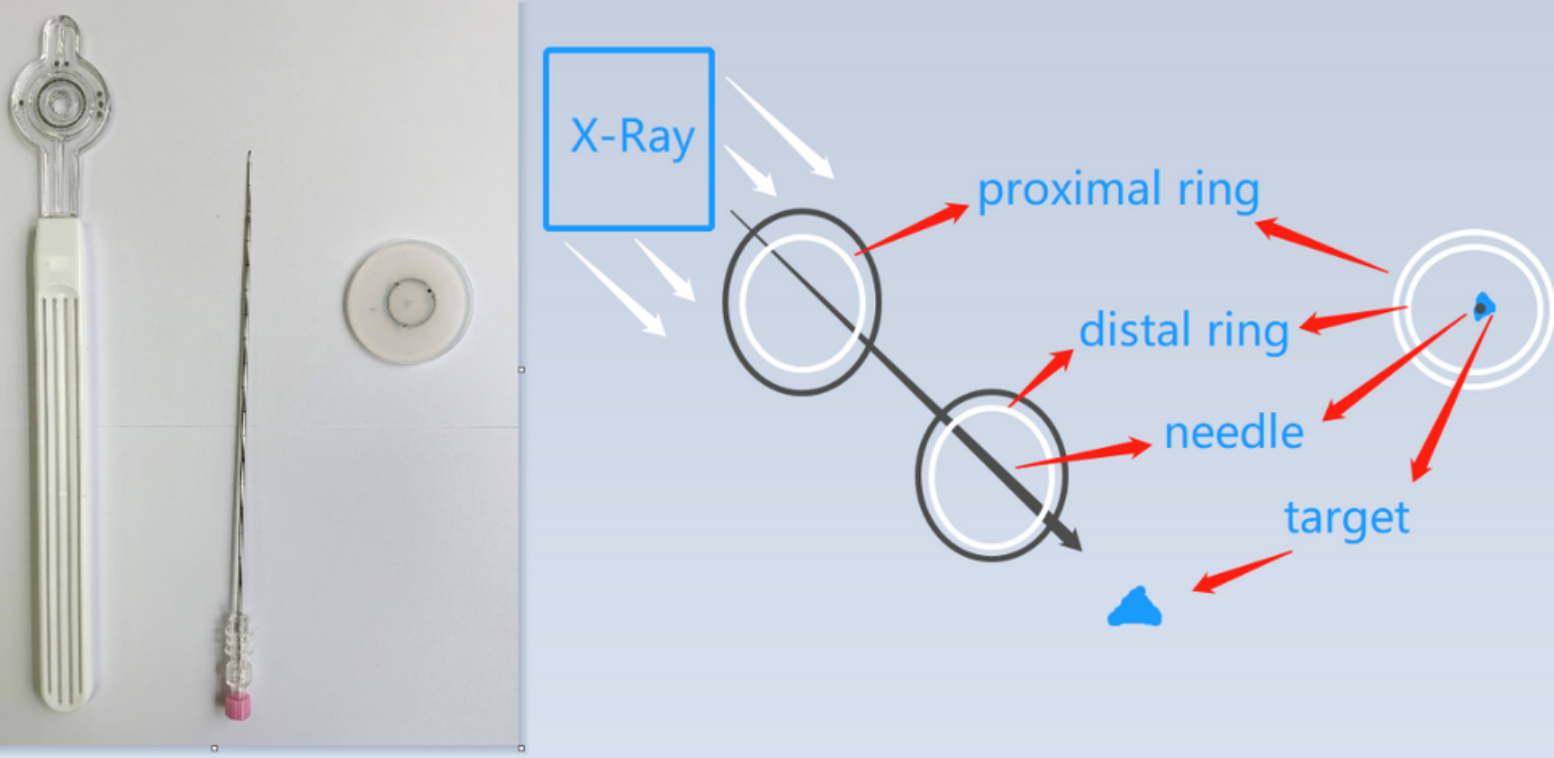

Figure 2

Two circular radiographic grids with handle. The central aperture of the proximal grid is constructed with a luer lock mechanism to allow the puncture needle to be detachably secured to the proximal grid.

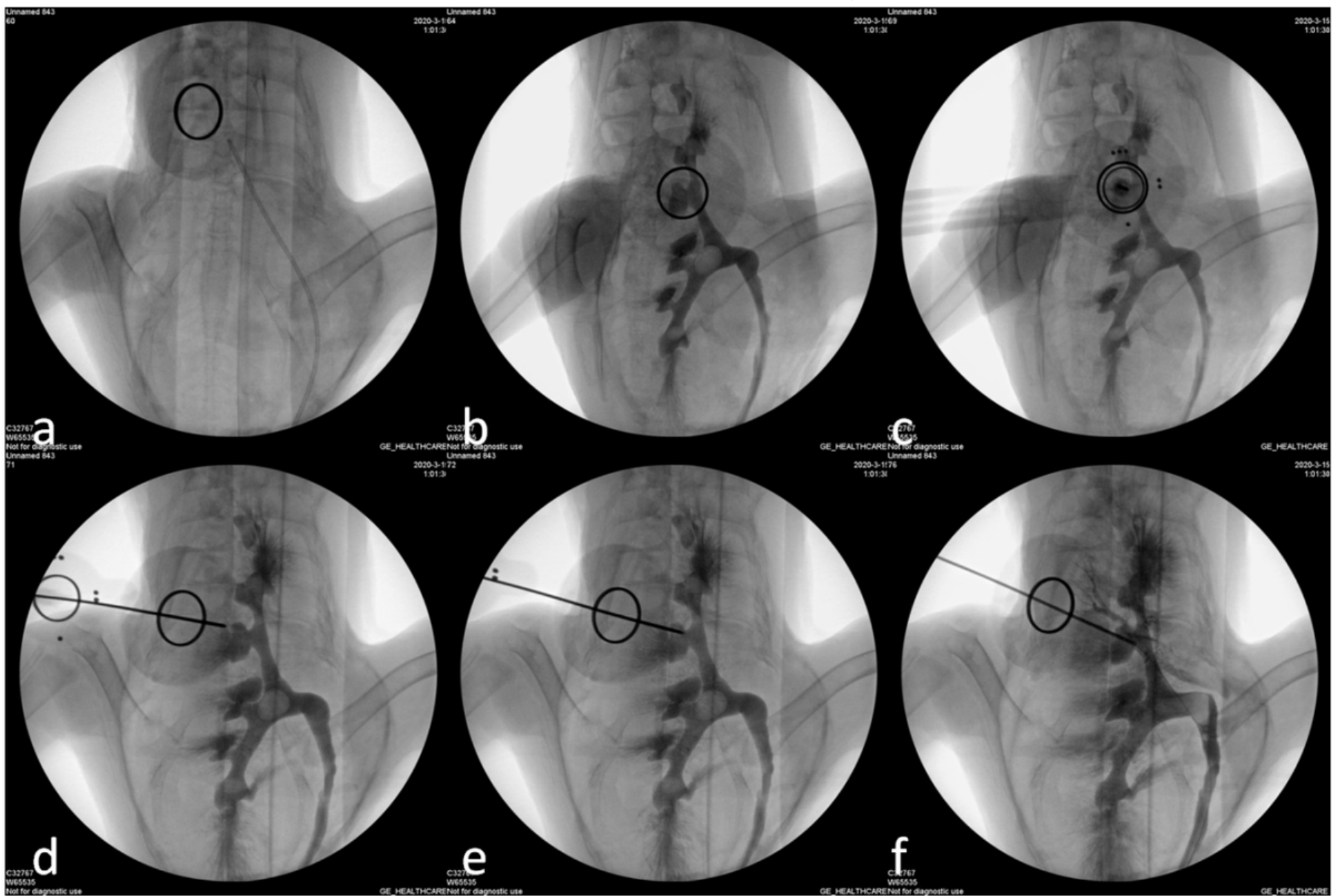

Figure 3 
Procedure images: (b) Select the target calyx with C-arm in $30^{\circ}$ angle (c) Adjust the two grids to superimpose the aiming rings (d) The $\mathrm{C}$-arm is rotated back to $0^{\circ}$ position to monitor the puncture depth. (e) The puncture needle enters the target renal. 